\title{
MiR-221 and miR-222 target PUMA to induce cell survival in glioblastoma
}

\author{
Chun-Zhi Zhang ${ }^{1,2,3+}$, Jun-Xia Zhang ${ }^{1,2+}$, An-Ling Zhang ${ }^{2,4 \dagger}$, Zhen-Dong Shi ${ }^{1,2+}$, Lei Han ${ }^{1,2}$, Zhi-Fan Jia ${ }^{2,4}$, \\ Wei-Dong Yang ${ }^{1}$, Guang-Xiu Wang ${ }^{2}$, Tao Jiang ${ }^{5}$, Yong-Ping You ${ }^{6}$, Pei-Yu Pu ${ }^{1,2}$, Jin-Quan Cheng ${ }^{7 *}$, \\ Chun-Sheng Kang ${ }^{1,2,4^{*}}$
}

\begin{abstract}
Background: MiR-221 and miR-222 (miR-221/222) are frequently up-regulated in various types of human malignancy including glioblastoma. Recent studies have reported that miR-221/222 regulate cell growth and cell cycle progression by targeting p27 and p57. However the underlying mechanism involved in cell survival modulation of miR-221/222 remains elusive.

Results: Here we showed that miR-221/222 inhibited cell apoptosis by targeting pro-apoptotic gene PUMA in human glioma cells. Enforced expression of miR-22/222 induced cell survival whereas knockdown of miR-221/222 rendered cells to apoptosis. Further, miR-221/222 reduced PUMA protein levels by targeting PUMA-3'UTR. Introducing PUMA cDNA without 3'UTR abrogated miR-221/222-induced cell survival. Notably, knockdown of miR221/222 induces PUMA expression and cell apoptosis and considerably decreases tumor growth in xenograft model. Finally, there was an inverse relationship between PUMA and miR-221/222 expression in glioma tissues.

Conclusion: To our knowledge, these data indicate for the first time that miR-221/222 directly regulate apoptosis by targeting PUMA in glioblastoma and that miR-221/222 could be potential therapeutic targets for glioblastoma intervention.
\end{abstract}

\section{Background}

MicroRNAs (miRNAs), a new class of small RNA ( 22nt), are thought to negatively regulate protein-coding genes by base-pair matching with 3'UTR of mRNA. Growing evidence has indicated that the important roles for miRNAs in the development of different cancers. Deregulation of miRNAs has been observed in various types of human malignancy, including lymphoma, colorectal cancer, lung cancer, breast cancer, papillary thyroid carcinoma, hepatocellular carcinoma and glioblastoma [1-7]. And oncomiRs and tumor suppressor miRNAs exert their functions through regulation of tumor suppressor genes and oncogenes, respectively [8-10].

\footnotetext{
* Correspondence: jin.cheng@moffitt.org; kang97061@yahoo.com

+ Contributed equally

'Department of Neurosurgery, Tianjin Medical University General Hospital,

Tianjin 300052, China

7 Departments of Molecular Oncology, H. Lee Moffitt Cancer Center and

Research Institute, Tampa, FL 33612, USA

Full list of author information is available at the end of the article
}

Glioblastoma is one of the most common forms of neural malignancy, with a median survival of 912 months. Despite the significant toxicities of current therapies, a large fraction of brain cancer patients suffer tumor recurrence due to the resistance to chemo- and radio-therapy [11-13]. Thus, there are urgent needs to develop novel therapeutic approaches by targeting the molecules that are altered in this malignancy. Recent studies showed frequent deregulation of miR-221/222 in glioblastoma $[1,14]$. We profiled miRNA expression in five glioblastoma cell lines, one astrocytoma cell line and one normal brain tissue, and found that miR-221/ 222 were overexpressed with a greater than 2 -fold increase in all glioma cell lines [15]. We and others have shown that miR-221/222 induce cell growth and cell cycle progression through negative regulation of $\mathrm{p} 27$ and p57 [16-19]. In the current study, we have demonstrated that miR-221/222 are of important role in regulation of cell apoptosis by direct targeting pro-apoptotic molecule PUMA in cell culture and xenograft model. Knockdown of miR-221/222 induces apoptosis and 
reduces tumor growth as well as upreulates PUMA expression. Whereas, ectopic expression of miR-221/222 exhibits opposite effects. Moreover, miR-221/222 directly interact with 3 'UTR of PUMA to repress PUMA expression. These findings indicate that PUMA is a bona fide target of miR-221/222 and these 2 miRNAs could be critical therapeutic targets for glioblastoma intervention.

\section{Results}

\section{Critical role of miR-221/222 in apoptosis pathway}

Previous studies have documented miR-221 and miR222 regulation of cell cycle progression and cell proliferation by targeting p27 and p57 [16-18]. However, their role in apoptotic pathway has not been well studied. As initial step, we carried out Northern blot analysis of miR-221 and miR-222 expression in a panel of glioma cell lines. Fig. 1A showed that U251, TJ866 and T)899 cells expressed higher levels of miR-221 and miR222 compared to other cell lines. To examine biological significance of miR-221 and miR-222 in glioma, U251 and LN229 cells were treated with As-miR-221 and/or As-miR-222 (Fig. 1B). Interestingly, annexin V-labeling revealed that knockdown of miR-221 and miR-222 significantly increased cell apoptosis compared to the cells treated with scramble oligonucleotide (Fig. 1C). Moreover, Western blot assay displayed that pro-apoptotic protein Bax expression was significantly up-regulated while BCL2 expression was down-regulated in As-miR221/222 group (Fig. 1D). In addition, caspase 3/7 activity was also considerably elevated in miR-221 and miR-222 knocked down cells (Fig. 1E). Since collapse of the mitochondrial membrane potential is one of the early events in apoptosis [20], we next examined if miR-221 and miR-222 regulate mitochondrial membrane potential. The cells with depletion of miR-221 and miR-222 were stained with cationic dye JC-1. FACSCalibur analysis showed that the mitochondrial membrane potential was largely damaged when miR-221 and miR-222 were depleted (Fig. 1F). These findings indicate that miR-221 and miR-222 play an important role in initiation of cell apoptosis.

\section{PUMA is a target for miR-221 and miR-222}

To determine the mechanism by which miR-221 and miR-222 regulate cell apoptosis, we performed miRNA target search using Pictar and found 3'UTR of PUMA containing the highly conserved putative miR-221 and miR-222 binding sites (Fig. 2A). Further, we knockeddown miR-221/222 in U251 cells, which exhibit elevated level of miR-221/222, and ectopically expressed miR221/222 in H4 cells with low endogenous miR-221/222 expression (Fig. 1A). Western blot analysis showed that PUMA expression was up-regulated in U251 cells with knockdown of miR-221/222 (Fig. 2B), whereas downregulated in $\mathrm{H} 4$ cells overexpressing miR-221/222 (Fig. 2C), compared to the cell treated with scrambled oligonucleotide or vector alone. Moreover, we created pGL3-WT-PUMA-3'UTR and pGL3-MUT-PUMA-3' UTR plasmids. Reporter assay revealed reduction of miR-221/222 led to a marked increase of luciferase activity of pGL3-WT-PUMA-3'UTR plasmid, without change in luciferase activity of pGL3-MUT-PUMA-3' UTR plasmid (Fig. 2D). PUMA recently identified as a critical mediator of p53-associated apoptosis. Thus, we further explored the effect of miR-221/222 on p53 expression. There was no change of p53 in U251 and LN229 cells after reduction of miR-221/222 (Fig. 2E). These indicate that miR-221/222 directly modulate PUMA expression by binding 3'UTR of PUMA.

\section{Expression of PUMA overrides miR-221/222 survival function}

Having demonstrated PUMA as a direct target of miR221 and miR-222, we next examined the importance of PUMA in miR-221/222-mediated cell survival. Since H4 expressed low level of miR-221/222, we transfected PUMA lacking 3 'UTR together with and without miR221 and miR-222 into the H4 cells (Fig. 3A). Annexin V analysis showed that expression of miR-221 and/or miR222 significantly reduced cell apoptosis induced by serum starvation (Fig. 3B). However, expression of PUMA largely abrogated miR-221/222 effects on cell apoptosis (Fig. 3C), suggesting that PUMA is a critical target of miR-221 and miR-222 involved in cell apoptosis.

\section{As-miR-221/222 inhibits glioblastoma xenograft growth accompanying PUMA upregulation}

Since miR-221 and miR-222 are frequently elevated in glioblastoma and play an important role in cell survival, we further examined the effects of knockdown of miR221/222 on tumor growth. As shown in Fig. 4A, tumors continued growing in both scramble and control groups. However, As-miR-221/222 significantly reduced tumor growth $(\mathrm{p}<0.05)$. After 5 days of withdraw of the treatment, tumor started to regrow. TUNEL assay analysis of xenograft tumor taken at 28 days after treatment revealed much more apoptosis in As-miR221/222 group when compared to tumors from scramble and control groups (Fig. 4B). We also histologically observed that there were more neonatal micro-vessels, bigger tumor cell nuclei, chromosomes stained a deeper blue, more mitotic tumor cells and fewer necrotic foci in control and scramble groups than those in As-miR221/222 group. Ki-67 staining shows that As-miR-221/222 treated tumors had a lower proliferation index compared with the control groups (Fig. 4C). In addition, LNA-ISH 


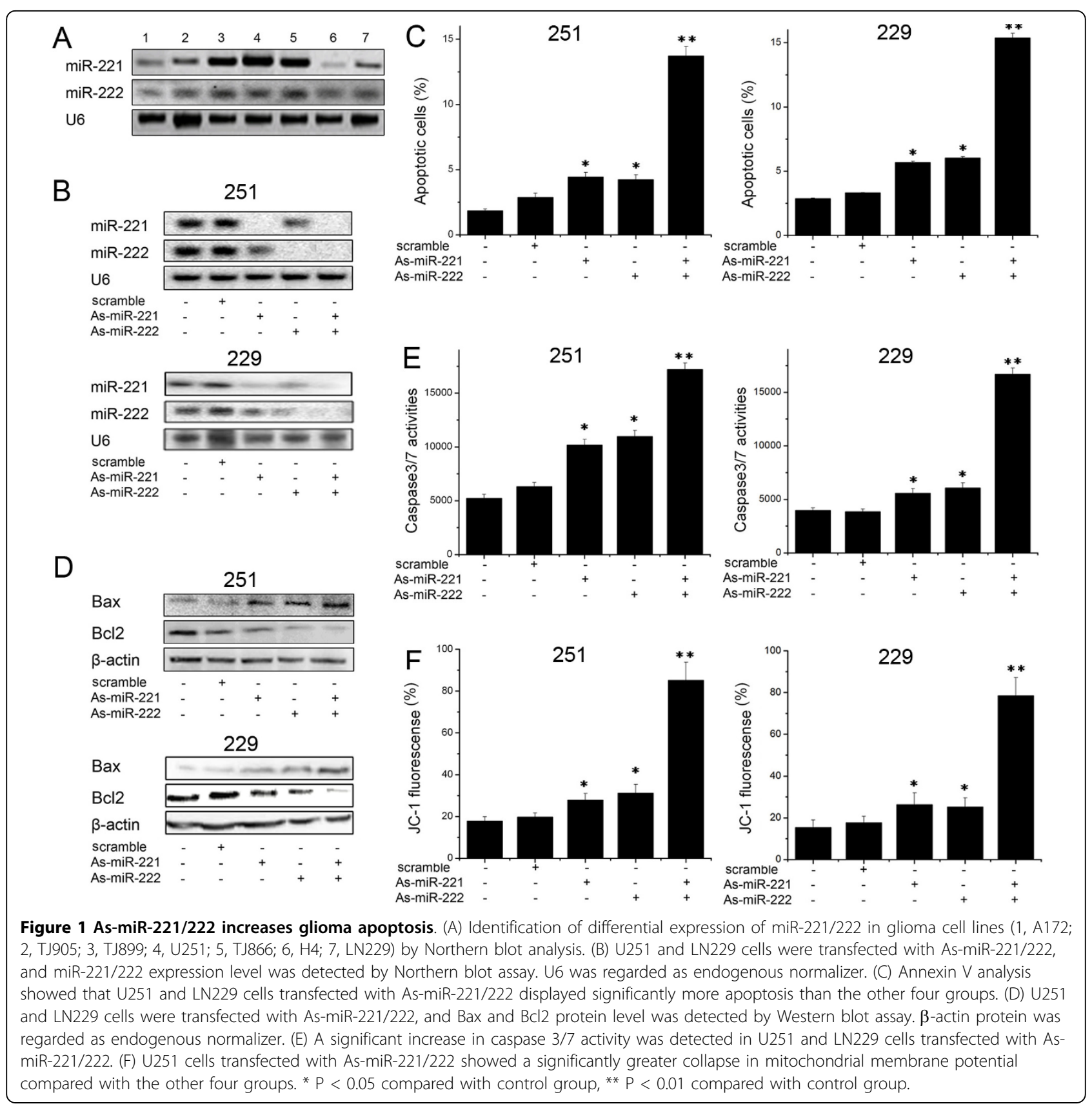

analysis confirmed that miR-221/222 levels were considerably reduced in As-miR221/222 group (Fig. 4D). Immunohistochemical stanining analysis revealed that PUMA levels were up-regulated in As-miR221/222 group (Fig. 4E), confirming the data in vitro that PUMA as a direct target of miR-221/222. Additionally, Bax expression was increased, whereas $\mathrm{Bcl} 2$ expression was decreased in xenograft tumor sections (Fig. 4G, F). These findings further indicate that miR-221/222 targets PUMA and that As-miR-221/222 could be therapeutic means for glioblastoma intervention.

\section{Inverse correlation of expression of $\mathrm{miR}-221 / 222$ and} PUMA in glioma tissues

Having demonstrated PUMA as a major target of miR$221 / 222$, we further investigated the correlation of between miR-221/222 and PUMA expression in gliomas. We examined 40 human glioma specimens with LNAISH and immunohistochemical staining. Representative images of miR-221/222 and PUMA were shown in Fig. 5A. Upregulation of miR-221/222 was detected in 26 gliomas (Fig. 5B). Of the 26 tumors with elevated miR221/222, 21 (81\%) had low levels of PUMA $(\mathrm{P}<0.001)$. 


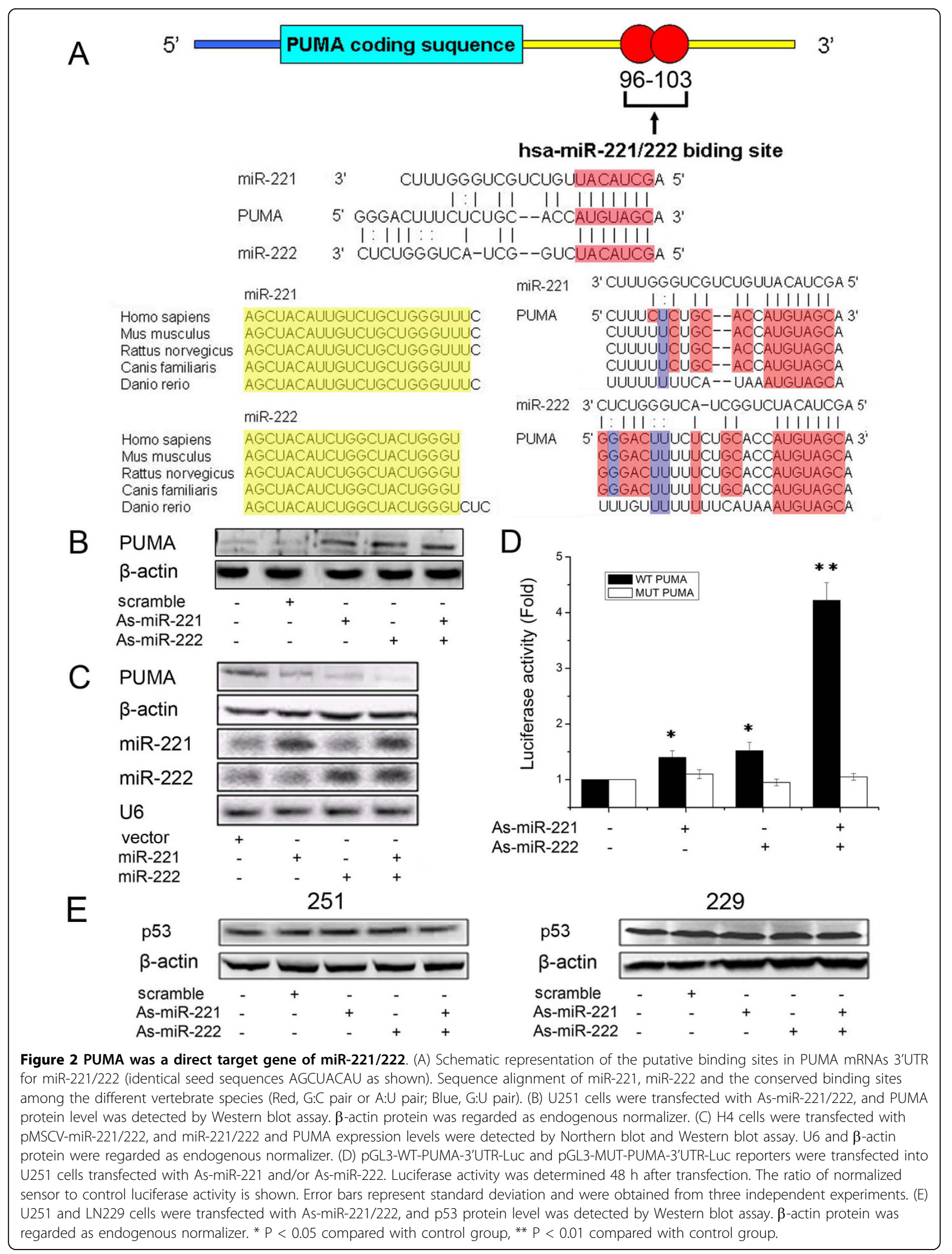


A

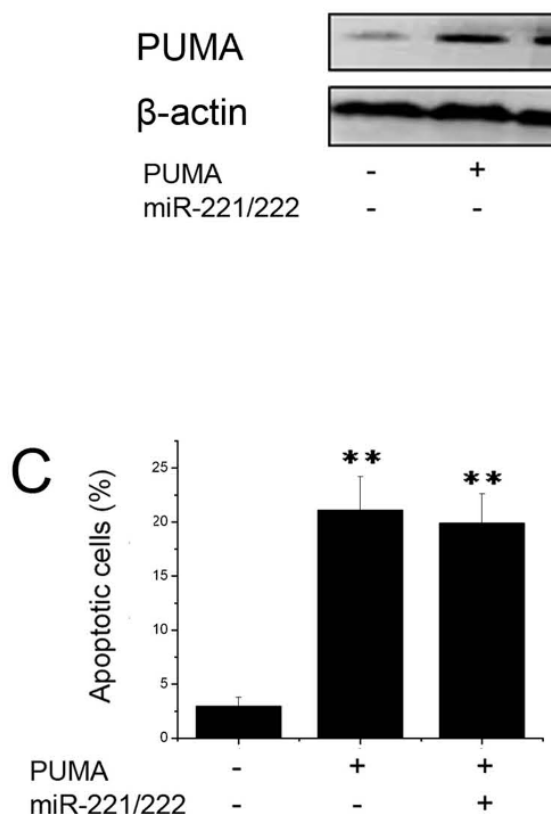$$
\text { IIR:221202 }
$$

$\mathrm{B}$

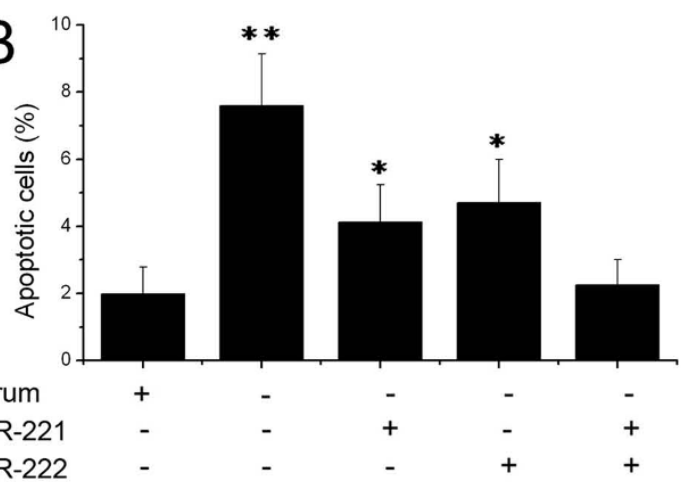

Figure 3 Expression of PUMA abrogates miR-221/222 survival function. (A) H4 cells were transfected pcDNA-PUMA (not including the 3'UTR), and PUMA expression was measured by Western blot assay. $\boldsymbol{\beta}$-actin protein was regarded as endogenous normalizer. (B) H4 cells were transfected with pMSCV-miR-221/222, then treated with serum starvation and cell apoptosis was Annexin $V$ analysis. (C) H4 cells were transfected with pcDNA-PUMA (not including the 3'UTR) and pMSCV-miR-221/222, and cell apoptosis, caspase activity and mitochondrial membrane potential were measured by Annexin $V$ analysis, caspase 3/7 activity assay and JC-1 staining. * $P<0.05$ compared with control group, ** $P<$ 0.01 compared with control group.

11 of 14 (79\%) specimens with downregulated miR-221/ 222 presented high levels of PUMA. In addition, we found that miR-221/222 expression increased significantly in high grade gliomas compared with low grade gliomas.

\section{Discussion}

Previous studies have shown frequent upregulation of miR-221/222 in human malignancies incuding glioblastoma [15]. MiR-221/222 regulate cell cycle through targeting p27 and p57 [16-19]. In the present study, we demonstrated the role of miR-221/222 in regulation of cell apoptosis. Knockdown of miR-221/222 induced change of mitochondrial membrane potential and caspase-mediated apoptosis. Further, we proved that proapoptotic protein PUMA was negatively regulated by miR-221/222. In addition, local injection of As-miR-221/ 222 reduced tumor growth and induced apoptosis in xenograft model. These findings provide the evidence of direct regulation of mitochondrial apoptotic pathway by $\mathrm{miR}-221 / 222$.

A mitochondrial-dependent step in apoptosis, involving mitochondrial outer membrane permeabilization (MOMP), is associated with most pro-apoptotic stimuli. This process is controlled by both pro- and anti- apoptotic members of the $\mathrm{Bcl} 2$ family and leads to the release of mitochondrial apoptotic factors such as cytochrome c, Smac/DIABLO and Omi/HtrA2 into the cytosol. Pro-apoptotic protein Bax induces a selective process of MOMP through the formation of channels or pores after oligomerization, allowing the release of proteins localized within the intermembrane space such as cytochrome c, which triggers caspase $9 / 3$ activation to induce the apoptotic phenotype [21]. However, Willis had detected no association of PUMA with Bax [22]. Other studies identified that PUMA indirectly activated Bax by binding and inactivating anti-apoptotic $\mathrm{Bcl} 2$ family members that include $\mathrm{Bcl} 2$ protein $[23,24]$. In our study, we found that knockdown miR-221/222 could downregulate $\mathrm{Bcl} 2$ and upregulate $\mathrm{Bax}$ by western blot assay. In addition, the change of BAX and Bcl2 expression in xenograft study with U251 cells confirmed the data in vitro. These results indicate that $\mathrm{miR}-221 / 222$ negatively regulate PUMA which leads to decrease Bcl2 and increase BAX.

Our results proved that modulating effect of miR-221/ 222 on PUMA by directly targeting PUMA. Bioinformatics analysis showed that 3'UTR of PUMA mRNA existed the highly conserved putative miR-221/222 binding sites. Luciferase reporter assay validated that PUMA 


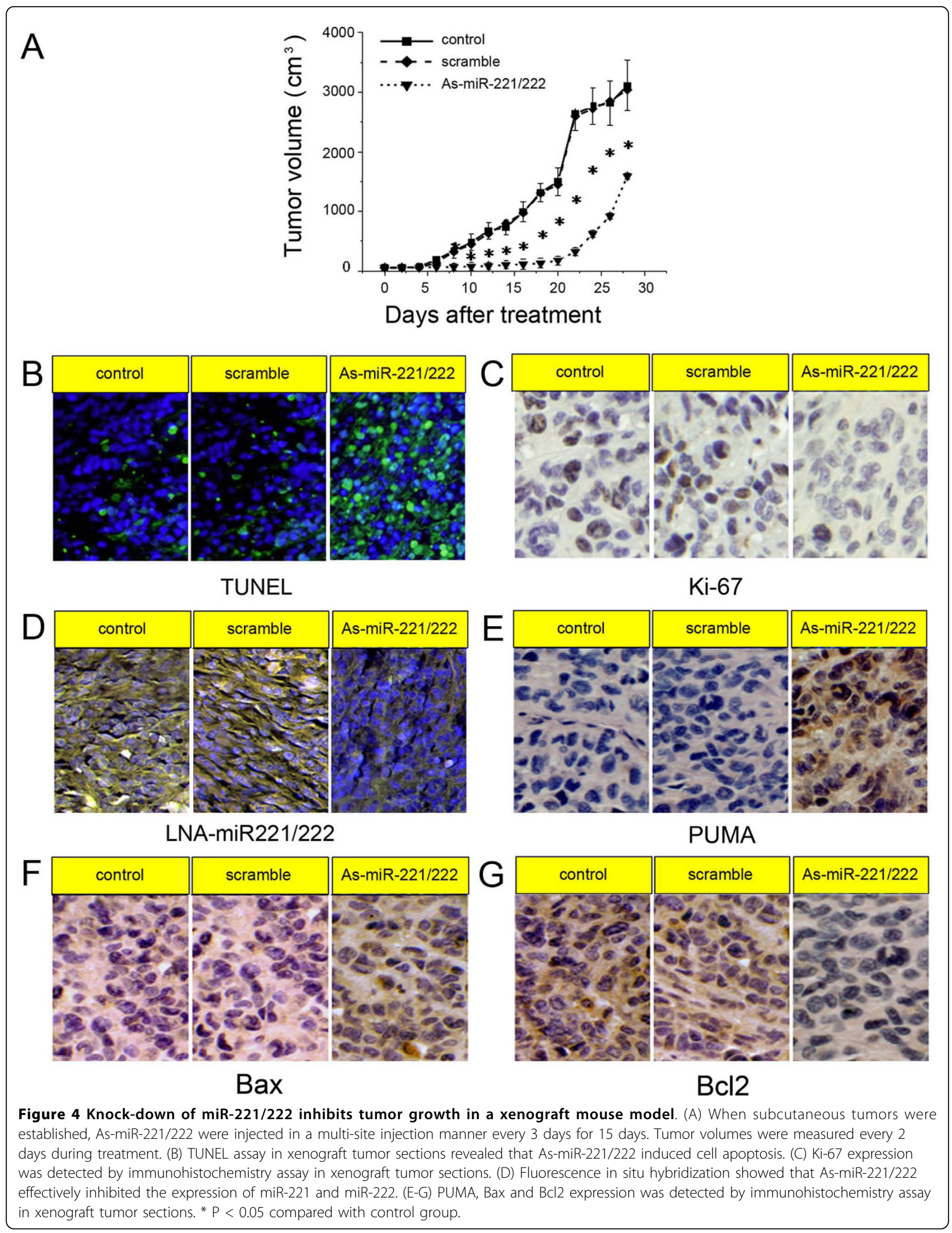




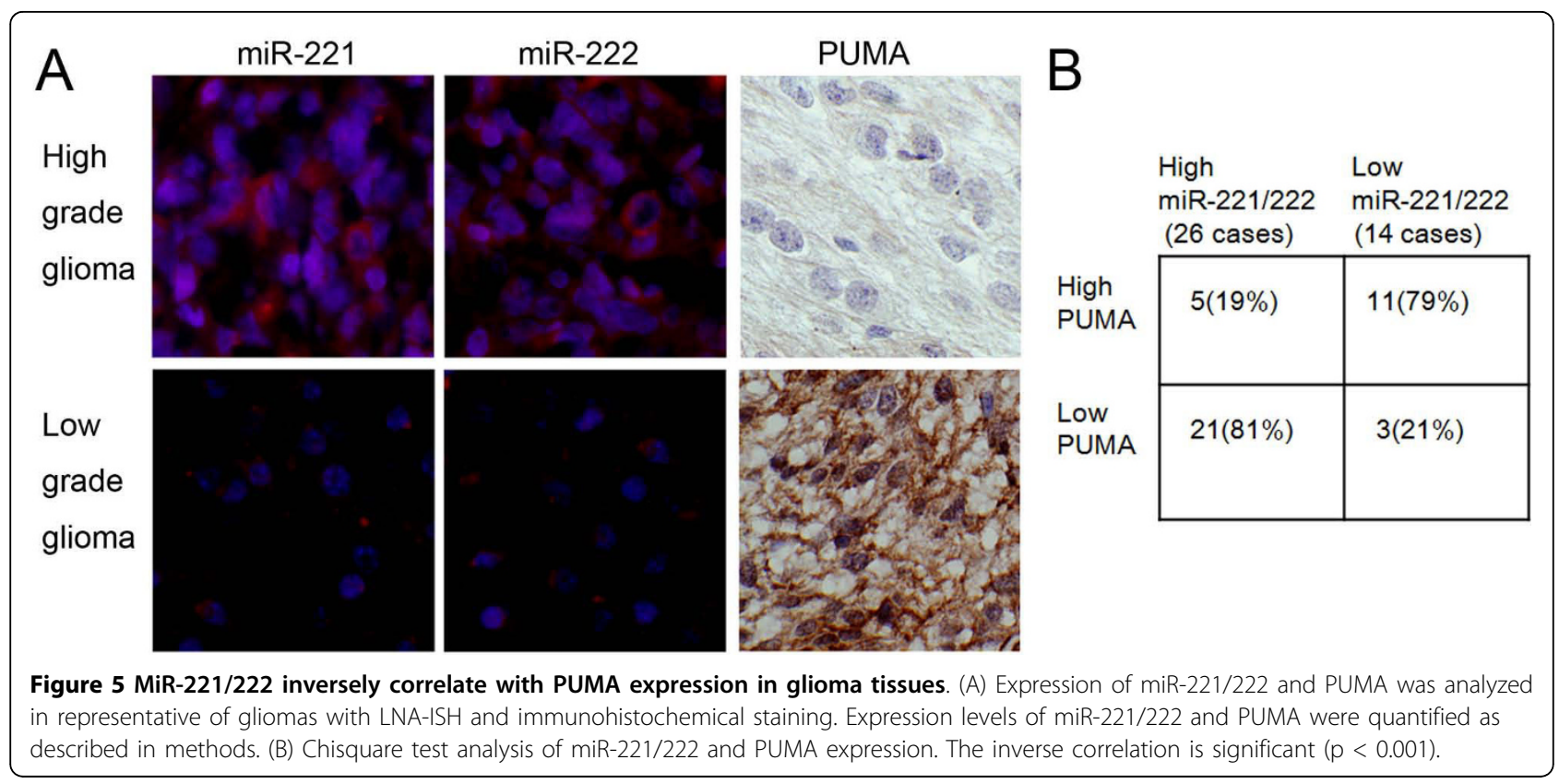

was a direct target of miR-221/222. Our xenograft study with U251 cells also shows that As-miR-221/222 treatment reduces tumor growth accompanying increase of PUMA expression and apoptosis. Further, there was an inverse relationship between PUMA and miR-221/222 expression levels in glioma tissues. PUMA, as a key mediator of p53-associated apoptosis, actually accounts for nearly all of the apoptotic activity attributed to p53. However, the expression level of p53 remained unchanged after reduction of miR-221/222. Thus, these finding suggest that PUMA is a core target of miR-221/ 222 in cell apoptosis.

\section{Conclusions}

In summary, our data demonstrated that miR-221/222 induced cell survival by direct targeting PUMA, and thus regulates mitochondrial pathway. We also provided direct evidence using As-miR-221/222 as therapeutic approaches for glioblastoma. The role of miR-221/222 in gilomagensis needs to be further investigated by creating transgenic mouse model.

\section{Methods}

\section{Cell culture and transfection}

Human glioblastoma cells (A172, U251, H4, LN229), retrovirus-packaging cells PT67 and the mouse fibroblast cell line NIH3T3 were obtained from the China Academia Sinica Cell Repository, Shanghai, China. Human glioblastoma cells (TJ905, TJ899, TJ866) were established in our lab. The cells were maintained in Dulbecco's modified Eagle's medium (DMEM) (Gibco) supplemented with $10 \%$ fetal bovine serum (Gibco), $2 \mathrm{mM}$ glutamine (Sigma), $100 \mu \mathrm{g} / \mathrm{ml}$ penicillin (Sigma), and $100 \mu \mathrm{g} / \mathrm{ml}$ streptomycin (Sigma), and incubated at $37^{\circ} \mathrm{C}$ with $5 \% \mathrm{CO}_{2}$. Cell transfection were used Lipofectamine 2000 (Invitrogen) following manufacture instruction.

\section{Plasmids, virus production and oligonucleotides}

Human pMSCV-miR-221 and -miR-222 were kindly provided by. R. Agami (The Netherlands Cancer Institute, Amsterdam, The Netherlands). The retroviruses expressing miR-221 or miR-222 were obtained by transfection of pMSCV-miR-221 and -miR-222 into PT67 packaging cells and selected with blasticidin $S(10 \mu \mathrm{g} /$ $\mathrm{ml}$ ) for six weeks. The viruses were tittered in NIH3T3 cells. Once cells grew to $60 \%$ confluence, cells were infected with pMSCV-miR-221 and/or pMSCV-miR-222 at a multiplicity of infection (MOI) of 50. HA-tagged wild-type PUMA in pCDNA3 was obtained from B. Volgelstein [25]. pGL3-WT-PUMA-3'UTR-Luc reporter was created by ligation of PCR products of 3 'UTR of PUMA into the $\mathrm{XbaI}$ site of the pGL3 control vector (Promega, USA). The primers for PCR amplification are: PUMA-3' UTR-Forward: 5'-TCA TGA ATTC GCC CCT CCC ACC TCC TGA CAC CCT GGC CAG CGC GGG GGA CTT TCT CTG C and PUMA-3'UTR-Reverse: 5'-CGC CCC CGG GAC AGG CAG GGC TGG GAG TCC AGT ATG CTA CAT GGT GCA GAG AAA GTC CC-3'. pGL3-MUT-PUMA-3'UTR-Luc reporter was generated from pGL3-WT-PUMA-3'UTR-Luc reporter by deleting the binding site for miR-221/222. The 2'-OMe-oligonucleotides were chemically synthesized and purified by high-performance liquid chromatography by GenePharma Co., Ltd. (Shanghai, China). 
The sequences are: 2'-OMe-As-miR-221 (As-miR-221), 5'-AGCUACAUUGUCUGCUGGGUUUC-3'; 2'-OMeAs-miR-222 (As-miR-222), 5'-AGCUACAUCUGGCUACUGGGU-3'. 200 pmol As-miR-221 and/or As-miR-222 were transfected using Lipofectamine 2000 (Invitrogen). Cells transfected with scrambled 2'-OMe oligonucleotides (scramble) were used as control.

\section{RNA extraction and Northern blot analysis}

Total RNA was isolated from cells using TRIzol reagent (Invitrogen) as previously described [26]. For Northern blotting, total RNAs $(20 \mu \mathrm{g})$ were separated on $12 \%$ denaturing polyacrylamide gels, and then transferred to Hybond $\mathrm{N}+$ nylon membrane (Ambion). Following the UV cross-linked, he membrane was hybridized with digoxigenin (DIG)-labeled miR-221 and miR-222 probes overnight in a buffer containing $5 \times \mathrm{SSC}, 20 \mathrm{mmol} / \mathrm{L}$ $\mathrm{Na}_{2} \mathrm{HPO}_{4}(\mathrm{pH} \mathrm{7.2)}$, 7\% SDS, $1 \times$ Denhardt's solution and $0.2 \mathrm{mg} / \mathrm{mL}$ salmon sperm DNA. The membrane was washed with $1 \times \mathrm{SSC} / 1 \% \mathrm{SDS}$ at $50^{\circ} \mathrm{C}$. After equilibration in detection buffer, blots were detected with a DIG Luminescent Detection Kit (Roche, USA) and analyzed by GeneGenius.

\section{Western blot, miRNA locked nucleic acid (LNA) in situ hybridization, immunohistochemistry and luciferase reporter assay}

Western blot, miRNA-LNA in situ hybridization and immunohistochemistry were performed as previously described [27]. MiR-221 and miR-222-LNA oligonucleotides contained locked nucleic acids at five locations (underlined): 5'-GAA ACC CAGG CA-G ACA ATG TAG CT-3' (miR-221); 5'-GĀG A $\bar{C} C$ CĀG TAĞ CCA GAT GTA GCT-3' (miR-222). For reporter assay, cells were cultured in 96-well plates and transfected with pGL3PUMA-3'UTR-Luc, and As-miR-221 and/or As-miR-221. Following $48 \mathrm{~h}$ incubation, luciferase activity was measured using a dual-luciferase reporter system (Promega).

\section{Apoptosis}

$48 \mathrm{~h}$ after transfection, apoptosis in cultured cells was evaluated with annexin $\mathrm{V}$ labeling, caspase 3/7 activity and mitochondrial membrane potential. For the annexin $\mathrm{V}$ assay, an annexin V-FITC labeled Apoptosis Detection Kit (Abcam) was used according to the manufacturer's protocol. Caspase $3 / 7$ activity was measured using Caspase-Glo 3/7 reagent (Promega). Mitochondrial membrane potential was determined with cationic dye JC-1 (5,5',6,6'-tetrachloro-1,1',3,3'- tetraethylbenzimidazolylcarbocyanine-chloride/C25H27Cl3N4) staining [21]. Briefly cells were harvested and first stained with PI. Following wash twice with PBS, cells were incubated with $10 \mathrm{mg} / \mathrm{ml} \mathrm{JC}-1$ for $20 \mathrm{~min}$ at room temperature and then analyzed with FACSCalibur to detect green fluorescence at excitation/emission wavelengths of 485/ $530 \mathrm{~nm}$ and red fluorescence at excitation/emission wavelengths of $485 / 590 \mathrm{~nm}$. TUNEL assay was used to detect the apoptosis in tumor specimens and was performed as previously described [15].

\section{Nude Mouse Tumor Xenograft Model and As-miR-221/222 treatment}

U251 glioma cells were subcutaneously injected to 5 -week old female nude mice (Cancer Institute of The Chinese Academy of Medical Science). When the tumor volume reached $50 \mathrm{~mm}^{3}$, the mice were randomly divided into three groups (ten mice per group) which were treated with 200 pmol scramble oligo, As-miR-221 and As-miR-222 in $10 \mu \mathrm{l}$ Lipofectamine or PBS through local injection of xenograft tumor in multiple sites. The treatment was performed once every 3 days for 15 days. The tumor volume was measured with a caliper every 2 days, using the formula: volume $=$ length $\times$ width $^{2} / 2$.

\section{Patients and samples}

From 40 glioma patients, we obtained 40 primary tumor samples during surgical resection of the lesion. Immediately after surgery, samples were snap-frozen and stored in liquid nitrogen. Histological diagnosis and grading of tumors were carried out with WHO criteria (World Health Organization, 2007). A total of 18 tumors were classified as low-grade gliomas, 10 as anaplastic astrocytomas, and the remaining 12 as glioblastoma multiformes (GBMs). Then miRNA-LNA in situ hybridization and immunohistochemistry were performed. Sections with no labeling or with fewer than $5 \%$ labeled cells were scored as 0 . Sections were scored as a 1 with labeling of $5-30 \%$ of cells, as a 2 with 31 $70 \%$ of cells and as a 3 with labeling of $\geq 71 \%$. The staining intensity was scored similarly, with 0 used for negative staining, 1 for weakly positive, 2 for moderately positive and 3 for strongly positive. The scores for the percentage of positive tumor cells and for the staining intensity were added to generate an immunoreactive score for each specimen. The product of the quantity and intensity scores were calculated such that a final score of $0-1$ indicated negative expression (-), 2-3 indicated weak expression (+), 4-5 indicated moderate expression $(++)$ and 6 indicated strong expression $(+++)$. Each sample was examined separately and scored by two pathologists.

\section{Statistical Analysis}

Statistical evaluation for data analysis was determined by t test. Differences with $\mathrm{P}<0.05$ were considered statistically significant 


\section{Acknowledgements}

This work was supported by The China National Natural Scientific Fund (30772231 and 30901772), and The Program for New Century Excellent Talents in University (NCET-07-0615). The authors wish to thank Dr. R Agami, Division of Tumor Biology, The Netherlands Cancer Institute, Amsterdam, The Netherlands, for kindly providing the retroviral constructs of miR-221 and miR-222.

\section{Author details}

'Department of Neurosurgery, Tianjin Medical University General Hospital, Tianjin 300052, China. ${ }^{2}$ Laboratory of Neuro-Oncology, Tianjin Medical University General Hospital, Tianjin 300052, China. ${ }^{3}$ Department of Radiation Oncology, Tianjin Huanhu hospital, Tianjin 300060, China. ${ }^{4}$ Tianjin Key Laboratory of Nerve Injury, Variation and Regeneration, Tianjin 300052, China. ${ }^{5}$ Department of Neurosurgery, Tiantan Hospital, Capital Medical University, Beijing 100050 China. ${ }^{6}$ Department of Neurosurgery, The First Affiliated Hospital of Nanjing Medical University, Nanjing 210029, PR China. 7 Departments of Molecular Oncology, H. Lee Moffitt Cancer Center and Research Institute, Tampa, FL 33612, USA.

\section{Authors' contributions}

CZZ, ALZ and ZDS performed the experimental work. JXZ interpreted the data and helped to draft the manuscript. LH, ZFJ, GXW and TJ participated in the experiments. WDY and YPY analyzed data. PYP, JQC and CSK conceived of the study and participated in its design and coordination. All authors have read and approved the final manuscript.

\section{Competing interests}

The authors declare that they have no competing interests.

Received: 29 March 2010 Accepted: 2 September 2010

Published: 2 September 2010

\section{References}

1. Ciafre SA, Galardi S, Mangiola A, Ferracin M, Liu CG, Sabatino G, Negrini M, Maira G, Croce CM, Farace MG: Extensive modulation of a set of microRNAs in primary glioblastoma. Biochem Biophys Res Commun 2005, 334:1351-1358.

2. He H, Jazdzewski K, Li W, Liyanarachchi S, Nagy R, Volinia S, Calin GA, Liu CG, Franssila K, Suster $S$, et al: The role of microRNA genes in papillary thyroid carcinoma. Proc Natl Acad Sci USA 2005, 102:19075-19080.

3. Iorio MV, Ferracin M, Liu CG, Veronese A, Spizzo R, Sabbioni S, Magri E, Pedriali M, Fabbri M, Campiglio M, et al: MicroRNA gene expression deregulation in human breast cancer. Cancer Res 2005, 65:7065-7070.

4. Metzler M, Wilda M, Busch K, Viehmann S, Borkhardt A: High expression of precursor microRNA-155/BIC RNA in children with Burkitt lymphoma. Genes Chromosomes Cancer 2004, 39:167-169.

5. Michael MZ, SM OC, van Holst Pellekaan NG, Young GP, James RJ: Reduced accumulation of specific microRNAs in colorectal neoplasia. Mol Cancer Res 2003, 1:882-891.

6. Murakami Y, Yasuda T, Saigo K, Urashima T, Toyoda H, Okanoue T, Shimotohno K: Comprehensive analysis of microRNA expression patterns in hepatocellular carcinoma and non-tumorous tissues. Oncogene 2006, 25:2537-2545.

7. Takamizawa J, Konishi H, Yanagisawa K, Tomida S, Osada H, Endoh H, Harano T, Yatabe Y, Nagino M, Nimura Y, et al: Reduced expression of the let-7 microRNAs in human lung cancers in association with shortened postoperative survival. Cancer Res 2004, 64:3753-3756.

8. Calin GA, Croce CM: MicroRNA signatures in human cancers. Nat Rev Cancer 2006, 6:857-866

9. Chen CZ: MicroRNAs as oncogenes and tumor suppressors. N Engl J Med 2005, 353:1768-1771

10. Shi L, Cheng Z, Zhang J, Li R, Zhao P, Fu Z, You Y: hsa-mir-181a and hsamir-181b function as tumor suppressors in human glioma cells. Brain Res 2008, 1236:185-193.

11. Eyler CE, Foo WC, LaFiura KM, McLendon RE, Hjelmeland AB, Rich JN: Brain cancer stem cells display preferential sensitivity to Akt inhibition. Stem Cells 2008, 26:3027-3036.
12. Geiger GA, Fu W, Kao GD: Temozolomide-mediated radiosensitization of human glioma cells in a zebrafish embryonic system. Cancer Res 2008, 68:3396-3404

13. Zheng $Y$, Lin $L$, Zheng Z: TGF-alpha induces upregulation and nuclear translocation of Hes1 in glioma cell. Cell Biochem Funct 2008, 26:692-700

14. Zhang J, Han L, Ge Y, Zhou X, Zhang A, Zhang C, Zhong Y, You Y, Pu P, Kang C: miR-221/222 promote malignant progression of glioma through activation of the Akt pathway. Int J Oncol 36:913-920.

15. Zhou X, Ren Y, Moore L, Mei M, You Y, Xu P, Wang B, Wang G, Jia Z, Pu P, et al: Downregulation of miR-21 inhibits EGFR pathway and suppresses the growth of human glioblastoma cells independent of PTEN status. Lab Invest 90:144-155.

16. Medina R, Zaidi SK, Liu CG, Stein JL, van Wijnen AJ, Croce CM, Stein GS: MicroRNAs 221 and 222 bypass quiescence and compromise cell survival. Cancer Res 2008, 68:2773-2780.

17. le Sage C, Nagel R, Egan DA, Schrier M, Mesman E, Mangiola A, Anile C, Maira G, Mercatelli N, Ciafre SA, et al: Regulation of the p27(Kip1) tumor suppressor by miR-221 and miR-222 promotes cancer cell proliferation. Embo J 2007, 26:3699-3708

18. Zhang C, Kang C, You Y, Pu P, Yang W, Zhao P, Wang G, Zhang A, Jia Z Han L, Jiang H: Co-suppression of miR-221/222 cluster suppresses human glioma cell growth by targeting p27kip1 in vitro and in vivo. Int J Oncol 2009, 34:1653-1660.

19. Fornari F, Gramantieri L, Ferracin M, Veronese A, Sabbioni S, Calin GA, Grazi GL, Giovannini C, Croce CM, Bolondi L, Negrini M: MiR-221 controls CDKN1C/p57 and CDKN1B/p27 expression in human hepatocellular carcinoma. Oncogene 2008, 27:5651-5661.

20. Yee KS, Wilkinson S, James J, Ryan KM, Vousden KH: PUMA- and Baxinduced autophagy contributes to apoptosis. Cell Death Differ 2009, 16:1135-1145.

21. Arnoult D: Apoptosis-associated mitochondrial outer membrane permeabilization assays. Methods 2008, 44:229-234.

22. Willis SN, Fletcher J, Kaufmann T, van Delft MF, Chen L, Czabotar PE, lerino $\mathrm{H}$, Lee EF, Fairlie WD, Bouillet $\mathrm{P}$, et al: Apoptosis initiated when BH3 ligands engage multiple Bcl-2 homologs, not Bax or Bak. Science 2007, 315:856-859.

23. Adams JM, Cory S: The BCl-2 apoptotic switch in cancer development and therapy. Oncogene 2007, 26:1324-1337.

24. Jabbour AM, Heraud JE, Daunt CP, Kaufmann T, Sandow J, O'Reilly LA, Callus BA, Lopez A, Strasser A, Vaux DL, Ekert PG: Puma indirectly activates Bax to cause apoptosis in the absence of Bid or Bim. Cell Death Differ 2009, 16:555-563.

25. Yu J, Zhang L, Hwang PM, Kinzler KW, Vogelstein B: PUMA induces the rapid apoptosis of colorectal cancer cells. Mol Cell 2001, 7:673-682.

26. Zhao JJ, Lin J, Yang H, Kong W, He L, Ma X, Coppola D, Cheng JQ: MicroRNA-221/222 negatively regulates estrogen receptor alpha and is associated with tamoxifen resistance in breast cancer. J Biol Chem 2008, 283:31079-31086.

27. Yamamichi N, Shimomura R, Inada K, Sakurai K, Haraguchi T, Ozaki Y, Fujita S, Mizutani T, Furukawa C, Fujishiro M, et al: Locked nucleic acid in situ hybridization analysis of miR-21 expression during colorectal cancer development. Clin Cancer Res 2009, 15:4009-4016.

doi:10.1186/1476-4598-9-229

Cite this article as: Zhang et al:: MiR-221 and miR-222 target PUMA to induce cell survival in glioblastoma. Molecular Cancer 2010 9:229. 